\title{
Impact of Flyash on Soil physical Properties under Sunflower-Spinach- Sunflower Crop Rotation System in Central India
}

\author{
Nouraldin Almahdi Ibrahim Basha, Abhishek James*, Ram Bharose and P. Smriti Rao
}

Department of Environmental Sciences and NRM, SHUATS, India

*Corresponding author

\section{A B S T R A C T}

Keywords

Flyash, Sunflower,

Spinach, Soil

texture, Water

holding capability,

Density, Bulk

density, Porosity etc

Article Info

Accepted:

15 November 2018

Available Online:

10 December 2018
Fly ash has tremendous potential as a nutrient supplement and plays a favourable role in increasing growth performance. Fly ash constitutes above $70 \%$ of the whole amount of residues formed in power plants through combustion of coal. The addition of Fly ash in soil improves or changes various physical, chemical and biological characteristics of soil. Fly ash is used for the improvement of the soil texture, water holding capability, density, bulk density, porosity etc. by using in different ratio with soil. For the growth of plant and crop yield fly ash is used as ameliorate in acidic soils. An experiment was conducted during the years 2015-17 adjacent to Yamuna River in the south of Allahabad city, India to find the impact of flyash on Soil physical Properties under Sunflower-Spinach-Sunflower Crop Rotation System. Findings of experiment reveal that the percentages of porosity, soil water holding capacity were increased while soil bulk density, soil particle density, soil solid space decreased as the dose of flyash increases under both cropping system even though after harvesting of crops there is slight decrease in these value.

\section{Introduction}

India has an enormous coal diffidence of 211 billion tonnes, coal is one of the most widely used fossil fuels for the generating of power. However, ash content of $40 \%$ to $50 \%$ in Indian coal presents an intrinsic problem of ash dumping. Year 2012, 175 million tonnes or more than of fly ash are usually to be generated in the country. According to Ministry of Power, (Government of India) around 1800 million tonnes coal will be use every year foremost to generation of 600 million tonnes fly ash by 2031-2032. Fly ash is the waste product of burning materials. These light particle are flew above with reduce gases. Fly ash constitutes above 70\% of the whole amount of residues formed in power plants through combustion of coal. Since huge amount of fly ash is generated every year, an enormous convention of research requirements to be conducted to find out the possibility of its exploitation in cultivation, manufacture and other industries around $10 \%$ of the total fly ash were utilized, generated as against $3-5 \%$. Consequently fly ash is currently treated as a precious source for appropriate conventional avenues of fly ash management. According to the Central Electricity Authority (CEA) New Delhi, India In year 2010-2011, $90 \mathrm{coal} /$ lignite thermal power stations of assorted power utilities in 
the country by way of total installed capacity of about $83797 \mathrm{MW}$, the whole generated fly ash is 66.49 million tonne and utilized ash was 36.26 million tonne, which provides percentage exploitation is $54.23 \%$ Raj and Mohan (2014). In India unaccompanied, the generation rate of fly ash is more than 112 million tons annually and it also shows that the production as well as both fly ash and bottom ash, may possibly to exec 170 million tons per year with the year 2015 (Pandey et al., 2009; Pandey and Singh 2010). The management of this enormous amount of solid waste, at both regional as well as global stage is a most important alarm in favour of the present and coming future (Ahmaruzzaman 2010, Kishore et al., 2010). The elementary composition both nutrient as well as toxic elements, varies due to types and sources of used coal (Camberato et al., 1997).

Fly ash has tremendous potential as a nutrient supplement and plays a favourable role in increasing growth and yield of ground nut (Ray et al., 2003). Fly ash has similar physicochemical properties with soil. It can mix homogeneously and can improve agronomic properties of soil (Change et al., 1979). Fly ash is the treasure of trace elements. It makes the trace element readily avail- able to the crop when mixed with soil (Plank and Mortens, 1974). Auxin in- creased respiration rates are suggestive of parallel relationship of growth, respiratory activity and found to increase RNA synthesis in tissue of higher plants (Still and Pill, 2003). Use of fly ash ameliorates soil acidity for maximum uptake of trace elements from fly ash which acted as a reserve of trace element when mixed in soil. Fly ash helps to retain water in the soil and also helped $\mathrm{CO} 2$ evolution. The plant hormone Indole acetic acid and Gibberellic acid helped protein, oil synthesis and also increased respira- tion rate. Soil metabolic manners, performance of amylase invertase and protease, chlorophyll $\mathrm{a}$ and $\mathrm{b}$, carotenoid and protein content are augmented in fly ash amended soil (Bhandari, 2006; Bozkurt and Karacal, 2001; Chadha, 1998).

Fly ash is valuable materials for agriculture and a good soil ameliorate. The addition of Fly ash in soil improves or changes various physical, chemical and biological characteristics of soil. Fly ash is used for the improvement of the soil texture, water holding capability, density, $\mathrm{pH}$, bulk density, porosity etc. by using in different ratio with soil. For the growth of plant and crop yield fly ash is used as ameliorate in acidic soils. Fly ash is comprised mainly of silt and clay sized particles. It has the prospective if applied at high adequate rates to enduringly improve soil texture and enhance moisture holding capacity. Fly ash is used in clayey soil considerably reduced bulk density and WHC stuffing of gratis lime and augmented the content of fine sand which enhanced the soil consistency thereby the growth and yield performance of sunflower. Fly ash is mixed into soil for increasing soil porosity and soil drainage and mobility of nutrients.

\section{Materials and Methods}

The experiment was conducted during the years 2015-16 and 2016-17 in the Rabi season to study the Effect of flyash on soil health and quality sunflower and spinach crops under sunflower-spinach-sunflower crop rotation at Allahabad, India. The area is situated on the right bank adjacent to Yamuna river in the south of Allahabad city, which is located at $25^{\circ} 24^{\prime} 08.71 \mathrm{~N}$ latitude and $81^{\circ} 50^{\prime} 16.95^{\prime}$ ' $\mathrm{E}$ longitude and 98 meter above the sea level. All the facilities necessary for experimentation were made available from the department. The area of Allahabad District comes under subtropical and arid climate prevailing in the south East part of Uttar Pradesh with extremes in the temperature dropping to $5-6{ }^{0} \mathrm{C}$ in December and January and very hot in summer with temperature ranging between 46$47{ }^{0} \mathrm{C}$ in the month of May and June. The 
average rainfall is around $1013.4 \mathrm{~mm}$ with maximum concentration during July to September and occasional frost in winter and hot wind (loo) in summer.

\section{Characteristic properties of soil}

The soil of the experimental field is alluvial under the soil order inseptisol and suborder fluvents. The mechanical and chemical analysis of soil was done before start of the experiment in order to characterize the various soil properties.

\section{Soil sample preparation}

Before conducting the experiments soil sample were taken from different place of the experimental fields from the $0-15$, and $15-30$ depth. The samples collected were mixed again, portioning and sieving (by sieve of 2 $\mathrm{mm})$.

\section{Mechanical analysis}

The results of mechanical analysis of soil are given below. Mechanical analysis was done by "Bouyoucous hydrometer method" as described by Jackson (1957).

\section{Flyash analysis}

The flyash was brought from the IFFCO Phulpur. After homogenization and drying three portions was took, digested with a nitric perchloric solution and toxic element concentrations was determined by Atomic Absorption Spectrophotometer.

\section{Statistical analysis}

The experiment was conducted in $4 \times 4$ factorial design having four treatments and three replications. The data recorded during the course of investigation was subjected to statistical analysis as per method of "Analysis of variance" (Fisher 1950). The significant and non-significant of treatment affect was judged with the help of ' $F$ ' variance ratio test calculated ' $F$ ' at $5 \%$ level of significance. Either effects was considered to be significant or non-significant. The significant difference between the mean was tested against the critical difference at 5\% level of significance. For testing of hypothesis, the following ANOVA table is used.

\section{Results and Discussion}

\section{Porosity}

The tables of 1(a) and (b) and figures of 1(a), (b), (c) and (d) shows that the effect of flyash on porosity (\%) of post-harvest soil under sunflower-spinach-sunflower crop rotation at $0-15 \mathrm{~cm}$ and $15-30 \mathrm{~cm}$ soil depth was found satisfactory in both experimental years 201516 and 2016-17. The maximum porosity percentage $46.52(\%)$ and $47.22(\%)$ was found in $\mathrm{T}_{7}$ (Flyash @ 50 metric tons ha ${ }^{-1}+\mathrm{RDF}$ ) at $0-15 \mathrm{~cm}$ soil depth which was statistically at par with the $\mathrm{T}_{6}$ in 2015-16 and 2016-17 and minimum porosity percentage $42.99(\%)$ and $42.63(\%)$ was found in $\mathrm{T}_{1}$ (Control). It was found that the percentage of porosity was increased as the dose of flyash increases in each treatment and also found that the percentage of porosity were decreased after harvesting of each crop under crop rotation in both experimental years. The maximum porosity percentage $46.42(\%)$ and $46.55(\%)$ was found in $\mathrm{T}_{7}$ (Flyash @ 50 metric tons ha ${ }^{-}$ $\left.{ }^{1}+\mathrm{RDF}\right)$ at $15-30 \mathrm{~cm}$ soil depth which was statistically at par with the $T_{6}$ in 2015-16 and 2016-17 and minimum porosity percentage $42.91(\%)$ and $42.88(\%)$ was found in $\mathrm{T}_{1}$ (Control). It was found that the percentage of porosity was increased as the dose of flyash increases in each treatment and also found that the percentage of porosity were decreased after harvesting of each crop under crop rotation in both experimental years. The results showed that the porosity of soil increases with the increase doses of flyash 
upto $\mathrm{T}_{5}$ after that it started to decrease due to condition of the soil similar results obtained by Aitken et al., 1984; Pathan et al., 2001.

\section{Bulk density $\left(\mathrm{mg} / \mathrm{m}^{3}\right)$}

The tables of 2(a) and (b) and figures of 2(a), (b), (c) and (d) shows that the effect of flyash on soil bulk density $\left(\mathrm{mg} / \mathrm{m}^{3}\right)$ of post-harvest soil under sunflower-spinach-sunflower crop rotation at $0-15 \mathrm{~cm}$ and $15-30 \mathrm{~cm}$ soil depth was found satisfactory in both experimental years 2015-16 and 2016-17. The maximum soil bulk density $1.35\left(\mathrm{mg} / \mathrm{m}^{3}\right)$ and 1.33 $\left(\mathrm{mg} / \mathrm{m}^{3}\right)$ was found in $\mathrm{T}_{1}$ (Control) at $0-15 \mathrm{~cm}$ soil depth which was statistically at par with the $\mathrm{T}_{2}$ in 2015-16 and 2016-17 and minimum soil bulk density $1.11\left(\mathrm{mg} / \mathrm{m}^{3}\right)$ and 1.07 $\left(\mathrm{mg} / \mathrm{m}^{3}\right.$ ) was found in $\mathrm{T}_{7}$ (Flyash @ 50 metric tons $\left.\mathrm{ha}^{-1}+\mathrm{RDF}\right)$. It was found that the soil bulk density was decreased as the dose of flyash increases in each treatment and also decreases the soil bulk density after harvesting of each crop under crop rotation in both experimental years. The maximum soil bulk density $1.35\left(\mathrm{mg} / \mathrm{m}^{3}\right)$ and $1.34\left(\mathrm{mg} / \mathrm{m}^{3}\right)$ was found in $\mathrm{T}_{1}$ (Control) at $0-15 \mathrm{~cm}$ soil depth which was statistically at par with the $T_{2}$ in 2015-16 and 2016-17 and minimum soil bulk density $1.11\left(\mathrm{mg} / \mathrm{m}^{3}\right)$ and $1.10\left(\mathrm{mg} / \mathrm{m}^{3}\right)$ was found in $\mathrm{T}_{7}$ (Flyash @ 50 metric tons ha ${ }^{-1}+$ RDF). It was found that the soil bulk density was decreased as the dose of flyash increases in each treatment and also decreases the soil bulk density after harvesting of each crop under crop rotation in both experimental years. The particle size range of fly-ash is similar to silt and changes the bulk density of soil. (Several experiments have been performed to measure the physical properties for a variety of soils mixed with up to $50 \%$ fly-ash (Chang et al., 1977; Jones and Amos, 1977) which reveals that soil fly-ash mixture tend to have lower bulk density than soil alone. The results revealed that the application of fly-ash at $0 \%, 5 \%, 10 \%, 20 \%, 30 \%, 40 \%$, and $50 \%$ by weight in soil significantly reduced the bulk density and improved the soil structure, similar results obtained by Garg et al., (2005) and Kene et al., (1991).

The tables of 3(a) and (b) and figures of 3(a), (b), (c) and (d) shows that the effect of flyash on soil particle density $\left(\mathrm{mg} / \mathrm{m}^{3}\right)$ of postharvest soil under sunflower-spinachsunflower crop rotation at 0-15 $\mathrm{cm}$ and 15-30 $\mathrm{cm}$ soil depth was found satisfactory in both experimental years 2015-16 and 2016-17. The maximum on soil particle density $3.22\left(\mathrm{mg} / \mathrm{m}^{3}\right)$ and $3.14\left(\mathrm{mg} / \mathrm{m}^{3}\right)$ was found in $\mathrm{T}_{1}$ (Control) at $0-15 \mathrm{~cm}$ soil depth which was statistically at par with the $T_{2}$ in 2015-16 and 2016-17 and minimum on soil particle density 1.11 $\left(\mathrm{mg} / \mathrm{m}^{3}\right)$ and $1.07\left(\mathrm{mg} / \mathrm{m}^{3}\right)$ was found in $\mathrm{T}_{7}$ (Flyash@50 metric tons ha ${ }^{-1}+$ RDF). It was found that the on soil particle density was decreased as the dose of flyash increases in each treatment and also decreases the on soil particle density after harvesting of each crop under crop rotation in both experimental years. The maximum on soil particle density $3.16\left(\mathrm{mg} / \mathrm{m}^{3}\right)$ and $3.22\left(\mathrm{mg} / \mathrm{m}^{3}\right)$ was found in $\mathrm{T}_{1}$ (Control) at $0-15 \mathrm{~cm}$ soil depth which was statistically at par with the $T_{2}$ in 2015-16 and 2016-17 and minimum on soil particle density $2.43\left(\mathrm{mg} / \mathrm{m}^{3}\right)$ and $2.44\left(\mathrm{mg} / \mathrm{m}^{3}\right)$ was found in $\mathrm{T}_{7}$ (Flyash@ @ 50 metric tons $\mathrm{ha}^{-1}+\mathrm{RDF}$ ). It was found that the on soil particle density was decreased as the dose of flyash increases in each treatment and also decreases the on soil particle density after harvesting of each crop under crop rotation in both experimental years.

\section{Solid space (\%)}

The tables of 3(a) and (b) and figures of 3(a), (b), (c) and (d) shows that the effect of flyash on soil solid space (\%) of post harvest soil under sunflower-spinach-sunflower crop rotation at $0-15 \mathrm{~cm}$ and $15-30 \mathrm{~cm}$ soil depth was found satisfactory in both experimental years 2015-16 and 2016-17. 
Table.1A Effect of Flyash on Porosity (\%) of Post-Harvest Soil under Sunflower Spinach Sunflower Crop rotation at $0-15 \mathrm{~cm}$ depth

\begin{tabular}{|c|c|c|c|c|c|c|c|c|}
\hline \multirow{2}{*}{ Treatment } & \multicolumn{9}{|c|}{$2015-16$} & \multicolumn{4}{c|}{ 2016-17 } \\
\cline { 2 - 10 } & Sunflower & Spinach & Sunflower & Mean & Sunflower & Spinach & Sunflower & Mean \\
\hline $\mathrm{T}_{1}$ & 45.36 & 42.79 & 40.83 & 42.99 & 44.84 & 42.61 & 40.43 & 42.63 \\
\hline $\mathrm{T}_{2}$ & 46.16 & 43.63 & 41.82 & 43.87 & 46.35 & 43.85 & 41.72 & 43.97 \\
\hline $\mathrm{T}_{3}$ & 46.38 & 44.59 & 42.46 & 44.48 & 46.83 & 44.81 & 42.42 & 44.69 \\
\hline $\mathrm{T}_{4}$ & 47.03 & 45.20 & 43.10 & 45.11 & 47.42 & 45.44 & 43.07 & 45.31 \\
\hline $\mathrm{T}_{5}$ & 47.25 & 45.93 & 43.82 & 45.67 & 47.93 & 46.16 & 43.89 & 45.99 \\
\hline $\mathrm{T}_{6}$ & 47.27 & 46.40 & 44.55 & 46.07 & 48.53 & 46.65 & 44.80 & 46.66 \\
\hline $\mathrm{T}_{7}$ & 47.15 & 47.07 & 45.33 & 46.52 & 48.76 & 47.32 & 45.57 & 47.22 \\
\hline F-test & $\mathbf{S}$ & $\mathbf{S}$ & $\mathrm{S}$ & $\mathbf{S}$ & $\mathbf{S}$ & $\mathbf{S}$ & $\mathrm{S}$ & $\mathbf{S}$ \\
\hline S. Ed. ( \pm ) & $\mathbf{0 . 3 1 9}$ & $\mathbf{0 . 2 1 0}$ & $\mathbf{0 . 2 4 3}$ & 0.26 & $\mathbf{0 . 5 4 8}$ & $\mathbf{0 . 9 1 1}$ & $\mathbf{0 . 2 1 3}$ & 0.56 \\
\hline C.D. at 5\% & $\mathbf{0 . 6 5 8}$ & $\mathbf{0 . 4 3 4}$ & $\mathbf{0 . 5 0 1}$ & 0.53 & $\mathbf{1 . 1 3 1}$ & $\mathbf{1 . 8 8 1}$ & $\mathbf{0 . 4 4 0}$ & 1.15 \\
\hline
\end{tabular}

Table.1B Effect of flyash on porosity (\%) of post-harvest soil under sunflower spinach sunflower crop rotation at $15-30 \mathrm{~cm}$ depth

\begin{tabular}{|c|c|c|c|c|c|c|c|c|}
\hline Treatment & \multicolumn{5}{|c|}{$2015-16$} & \multicolumn{4}{c|}{$2016-17$} \\
\hline & Sunflower & Spinach & Sunflower & Mean & Sunflower & Spinach & Sunflower & Mean \\
\hline $\mathrm{T}_{1}$ & 45.27 & 42.70 & 40.75 & 42.91 & 45.01 & 42.79 & 40.83 & 42.88 \\
\hline $\mathrm{T}_{2}$ & 45.94 & 43.55 & 41.63 & 43.71 & 45.66 & 43.63 & 41.82 & 43.70 \\
\hline $\mathrm{T}_{3}$ & 46.33 & 44.42 & 42.41 & 44.39 & 45.68 & 44.59 & 42.46 & 44.24 \\
\hline $\mathrm{T}_{4}$ & 46.83 & 45.12 & 42.81 & 44.92 & 46.53 & 45.20 & 43.10 & 44.94 \\
\hline $\mathrm{T}_{5}$ & 46.94 & 45.62 & 43.73 & 45.43 & 46.98 & 45.93 & 43.82 & 45.58 \\
\hline $\mathrm{T}_{6}$ & 47.20 & 46.28 & 44.50 & 45.99 & 47.11 & 46.40 & 44.55 & 46.02 \\
\hline $\mathrm{T}_{7}$ & 47.11 & 47.03 & 45.12 & 46.42 & 47.25 & 47.07 & 45.33 & 46.55 \\
\hline F-test & $\mathbf{S}$ & $\mathbf{S}$ & $\mathrm{S}$ & $\mathbf{S}$ & $\mathbf{S}$ & $\mathrm{S}$ & $\mathbf{S}$ & $\mathbf{S}$ \\
\hline S. Ed. $( \pm)$ & 0.367 & 0.224 & 0.209 & 0.27 & 0.500 & 0.210 & 0.243 & 0.32 \\
\hline C.D. at 5\% & 0.757 & 0.463 & 0.431 & 0.55 & 1.031 & 0.434 & 0.501 & 0.66 \\
\hline
\end{tabular}

Table.2A Effect of flyash on bulk density $\left(\mathrm{mg} / \mathrm{m}^{3}\right)$ of post-harvest soil under sunflower spinach sunflower crop rotation at $0-15 \mathrm{~cm}$ depth

\begin{tabular}{|c|c|c|c|c|c|c|c|c|}
\hline \multirow{2}{*}{ Treatment } & \multicolumn{9}{|c|}{$2015-16$} & \multicolumn{4}{c|}{ 2016-17 } \\
\cline { 2 - 10 } & Sunflower & Spinach & Sunflower & Mean & Sunflower & Spinach & Sunflower & Mean \\
\hline $\mathrm{T}_{1}$ & 1.37 & 1.35 & 1.32 & 1.35 & 1.35 & 1.33 & 1.31 & 1.33 \\
\hline $\mathrm{T}_{2}$ & 1.34 & 1.32 & 1.30 & 1.32 & 1.34 & 1.33 & 1.32 & 1.33 \\
\hline $\mathrm{T}_{3}$ & 1.31 & 1.26 & 1.21 & 1.26 & 1.31 & 1.28 & 1.28 & 1.29 \\
\hline $\mathrm{T}_{4}$ & 1.28 & 1.22 & 1.18 & 1.23 & 1.26 & 1.22 & 1.21 & 1.23 \\
\hline $\mathrm{T}_{5}$ & 1.24 & 1.18 & 1.12 & 1.18 & 1.18 & 1.18 & 1.15 & 1.17 \\
\hline $\mathrm{T}_{6}$ & 1.22 & 1.15 & 1.08 & 1.15 & 1.14 & 1.15 & 1.12 & 1.14 \\
\hline $\mathrm{T}_{7}$ & 1.19 & 1.11 & 1.02 & 1.11 & 1.11 & 1.10 & 0.99 & 1.07 \\
\hline F-test & $\mathbf{S}$ & $\mathbf{S}$ & $\mathrm{S}$ & $\mathbf{S}$ & $\mathbf{S}$ & $\mathrm{S}$ & $\mathbf{S}$ & $\mathbf{S}$ \\
\hline S. Ed. $( \pm)$ & $\mathbf{0 . 0 0 8}$ & $\mathbf{0 . 0 1 6}$ & $\mathbf{0 . 0 5 0}$ & 0.02 & $\mathbf{0 . 0 4 4}$ & $\mathbf{0 . 0 1 5}$ & $\mathbf{0 . 0 8 5}$ & 0.05 \\
\hline C.D. at 5\% & $\mathbf{0 . 0 1 6}$ & $\mathbf{0 . 0 3 3}$ & $\mathbf{0 . 1 0 3}$ & 0.05 & $\mathbf{0 . 0 9 1}$ & $\mathbf{0 . 0 3 0}$ & $\mathbf{0 . 1 7 6}$ & 0.10 \\
\hline
\end{tabular}


Table.2B Effect of flyash on bulk density $\left(\mathrm{mg} / \mathrm{m}^{3}\right)$ of post-harvest soil under sunflower spinach sunflower crop rotation at 15-30 cm depth

\begin{tabular}{|c|c|c|c|c|c|c|c|c|}
\hline Treatment & \multicolumn{9}{|c|}{$2015-16$} & \multicolumn{4}{c|}{ 2016-17 } \\
\hline & Sunflower & Spinach & Sunflower & Mean & Sunflower & Spinach & Sunflower & Mean \\
\hline $\mathrm{T}_{1}$ & 1.37 & 1.35 & 1.32 & 1.35 & 1.36 & 1.34 & 1.31 & 1.34 \\
\hline $\mathrm{T}_{2}$ & 1.34 & 1.32 & 1.30 & 1.32 & 1.33 & 1.31 & 1.29 & 1.31 \\
\hline $\mathrm{T}_{3}$ & 1.31 & 1.26 & 1.21 & 1.26 & 1.30 & 1.25 & 1.20 & 1.25 \\
\hline $\mathrm{T}_{4}$ & 1.28 & 1.22 & 1.18 & 1.23 & 1.27 & 1.21 & 1.17 & 1.22 \\
\hline $\mathrm{T}_{5}$ & 1.24 & 1.18 & 1.12 & 1.18 & 1.23 & 1.17 & 1.11 & 1.17 \\
\hline $\mathrm{T}_{6}$ & 1.22 & 1.15 & 1.08 & 1.15 & 1.21 & 1.15 & 1.06 & 1.14 \\
\hline $\mathrm{T}_{7}$ & 1.19 & 1.11 & 1.02 & 1.11 & 1.18 & 1.10 & 1.01 & 1.10 \\
\hline F-test & $\mathbf{S}$ & $\mathbf{S}$ & $\mathrm{S}$ & $\mathbf{S}$ & $\mathbf{S}$ & $\mathrm{S}$ & $\mathbf{S}$ & $\mathbf{S}$ \\
\hline S. Ed. $( \pm)$ & 0.008 & 0.016 & 0.050 & 0.02 & 0.008 & 0.017 & 0.050 & 0.03 \\
\hline C.D. at 5\% & 0.016 & 0.033 & 0.103 & 0.05 & 0.016 & 0.035 & 0.104 & 0.05 \\
\hline
\end{tabular}

Table.3A Effect of flyash on particle density $\left(\mathrm{mg} / \mathrm{m}^{3}\right)$ of post-harvest soil under sunflower spinach sunflower crop rotation at $0-15 \mathrm{~cm}$ depth

\begin{tabular}{|c|c|c|c|c|c|c|c|c|}
\hline \multirow{2}{*}{ Treatment } & \multicolumn{9}{|c|}{$2015-16$} & \multicolumn{4}{c|}{ 2016-17 } \\
\cline { 2 - 10 } & Sunflower & Spinach & Sunflower & Mean & Sunflower & Spinach & Sunflower & Mean \\
\hline $\mathrm{T}_{1}$ & 3.34 & 3.18 & 3.14 & 3.22 & 3.22 & 3.13 & 3.08 & 3.14 \\
\hline $\mathrm{T}_{2}$ & 3.19 & 3.09 & 2.99 & 3.09 & 3.32 & 3.11 & 2.93 & 3.12 \\
\hline $\mathrm{T}_{3}$ & 2.99 & 2.99 & 2.83 & 2.94 & 3.13 & 3.09 & 2.72 & 2.98 \\
\hline $\mathrm{T}_{4}$ & 2.88 & 2.86 & 2.65 & 2.80 & 2.83 & 2.79 & 2.54 & 2.72 \\
\hline $\mathrm{T}_{5}$ & 2.76 & 2.77 & 2.50 & 2.68 & 2.58 & 2.49 & 2.39 & 2.49 \\
\hline $\mathrm{T}_{6}$ & 2.71 & 2.65 & 2.33 & 2.56 & 2.35 & 2.31 & 2.19 & 2.28 \\
\hline $\mathrm{T}_{7}$ & 2.63 & 2.54 & 2.16 & 2.44 & 2.18 & 2.16 & 2.05 & 2.13 \\
\hline F-test & $\mathbf{S}$ & $\mathbf{S}$ & $\mathrm{S}$ & $\mathbf{S}$ & $\mathbf{S}$ & $\mathrm{S}$ & $\mathbf{S}$ & $\mathbf{S}$ \\
\hline S. Ed. $( \pm)$ & $\mathbf{0 . 0 5 2}$ & $\mathbf{0 . 0 4 0}$ & $\mathbf{0 . 2 0 0}$ & 0.10 & 0.175 & 0.143 & 0.188 & 0.17 \\
\hline C.D. at 5\% & $\mathbf{0 . 1 0 8}$ & $\mathbf{0 . 0 8 3}$ & $\mathbf{0 . 4 1 3}$ & 0.20 & 0.362 & 0.294 & 0.388 & 0.35 \\
\hline
\end{tabular}

Table.3B Effect of flyash on particle density $\left(\mathrm{mg} / \mathrm{m}^{3}\right)$ of post-harvest soil under sunflower spinach sunflower crop rotation at $15-30 \mathrm{~cm}$ depth

\begin{tabular}{|c|c|c|c|c|c|c|c|c|}
\hline Treatment & \multicolumn{9}{|c|}{$2015-16$} & \multicolumn{4}{c|}{ 2016-17 } \\
\hline & Sunflower & Spinach & Sunflower & Mean & Sunflower & Spinach & Sunflower & Mean \\
\hline $\mathrm{T}_{1}$ & 3.24 & 3.15 & 3.10 & 3.16 & 3.34 & 3.18 & 3.14 & 3.22 \\
\hline $\mathrm{T}_{2}$ & 3.16 & 2.93 & 2.90 & 3.00 & 3.19 & 3.09 & 2.99 & 3.09 \\
\hline $\mathrm{T}_{3}$ & 2.87 & 2.88 & 2.77 & 2.84 & 2.99 & 2.99 & 2.83 & 2.94 \\
\hline $\mathrm{T}_{4}$ & 2.77 & 2.67 & 2.62 & 2.69 & 2.88 & 2.86 & 2.65 & 2.80 \\
\hline $\mathrm{T}_{5}$ & 2.71 & 2.58 & 2.45 & 2.58 & 2.76 & 2.77 & 2.50 & 2.68 \\
\hline $\mathrm{T}_{6}$ & 2.69 & 2.55 & 2.31 & 2.52 & 2.71 & 2.65 & 2.33 & 2.56 \\
\hline $\mathrm{T}_{7}$ & 2.62 & 2.53 & 2.14 & 2.43 & 2.63 & 2.54 & 2.16 & 2.44 \\
\hline F-test & $\mathbf{S}$ & $\mathbf{S}$ & $\mathrm{S}$ & $\mathbf{S}$ & $\mathbf{S}$ & $\mathrm{S}$ & $\mathbf{S}$ & $\mathbf{S}$ \\
\hline S. Ed. $( \pm)$ & 0.096 & 0.114 & 0.183 & 0.13 & 0.052 & 0.040 & 0.200 & 0.10 \\
\hline C.D. at 5\% & 0.199 & 0.235 & 0.377 & 0.27 & 0.108 & 0.083 & 0.413 & 0.20 \\
\hline
\end{tabular}


Table.4A Effect of flyash on solid (\%) of post-harvest soil under sunflower spinach sunflower crop rotation at $0-15 \mathrm{~cm}$ depth

\begin{tabular}{|c|c|c|c|c|c|c|c|c|}
\hline Treatment & \multicolumn{5}{|c|}{$2015-16$} & \multicolumn{4}{c|}{$2016-17$} \\
& Sunflower & Spinach & Sunflower & Mean & Sunflower & Spinach & Sunflower & Mean \\
\hline $\mathrm{T}_{1}$ & 44.84 & 43.68 & 42.96 & 43.83 & 43.16 & 43.14 & 42.64 & 42.98 \\
\hline $\mathrm{T}_{2}$ & 44.85 & 42.93 & 42.39 & 43.39 & 44.51 & 42.55 & 42.36 & 43.14 \\
\hline $\mathrm{T}_{3}$ & 44.61 & 42.40 & 41.80 & 42.94 & 44.05 & 42.02 & 41.70 & 42.59 \\
\hline $\mathrm{T}_{4}$ & 44.45 & 41.53 & 40.95 & 42.31 & 43.86 & 41.96 & 41.10 & 42.31 \\
\hline $\mathrm{T}_{5}$ & 44.46 & 40.62 & 39.94 & 41.67 & 43.81 & 41.62 & 40.00 & 41.81 \\
\hline $\mathrm{T}_{6}$ & 44.19 & 39.74 & 39.05 & 40.99 & 43.46 & 41.00 & 39.34 & 41.27 \\
\hline $\mathrm{T}_{7}$ & 43.92 & 38.98 & 38.22 & 40.37 & 42.43 & 39.66 & 38.76 & 40.28 \\
\hline F-test & $\mathbf{S}$ & $\mathbf{S}$ & $\mathbf{S}$ & $\mathbf{S}$ & $\mathbf{S}$ & $\mathrm{S}$ & $\mathbf{S}$ & $\mathbf{S}$ \\
\hline S. Ed. $( \pm)$ & $\mathbf{0 . 1 8 4}$ & $\mathbf{0 . 6 5 7}$ & $\mathbf{0 . 6 3 3}$ & 0.49 & 0.488 & 0.595 & 0.317 & 0.47 \\
\hline C.D. at 5\% & $\mathbf{0 . 3 7 9}$ & $\mathbf{1 . 3 5 6}$ & $\mathbf{1 . 3 0 7}$ & 1.01 & 1.008 & 1.227 & 0.655 & 0.96 \\
\hline
\end{tabular}

Table.4B Effect of flyash on solid (\%) of post-harvest soil under sunflower spinach sunflower crop rotation at $15-30 \mathrm{~cm}$ depth

\begin{tabular}{|c|c|c|c|c|c|c|c|c|}
\hline Treatment & \multicolumn{5}{|c|}{$2015-16$} & \multicolumn{4}{c|}{$2016-17$} \\
\hline & Sunflower & Spinach & Sunflower & Mean & Sunflower & Spinach & Sunflower & Mean \\
\hline $\mathrm{T}_{1}$ & 44.84 & 43.24 & 42.44 & 43.51 & 44.84 & 43.68 & 42.96 & 43.83 \\
\hline $\mathrm{T}_{2}$ & 44.85 & 42.77 & 42.28 & 43.30 & 44.85 & 42.93 & 42.39 & 43.39 \\
\hline $\mathrm{T}_{3}$ & 44.61 & 42.06 & 41.62 & 42.76 & 44.61 & 42.40 & 41.80 & 42.94 \\
\hline $\mathrm{T}_{4}$ & 44.45 & 41.13 & 40.90 & 42.16 & 44.45 & 41.53 & 40.95 & 42.31 \\
\hline $\mathrm{T}_{5}$ & 44.46 & 40.22 & 39.75 & 41.48 & 44.46 & 40.62 & 39.94 & 41.67 \\
\hline $\mathrm{T}_{6}$ & 44.19 & 39.36 & 38.76 & 40.77 & 44.19 & 39.74 & 39.05 & 40.99 \\
\hline $\mathrm{T}_{7}$ & 43.92 & 38.79 & 38.13 & 40.28 & 43.92 & 38.98 & 38.22 & 40.37 \\
\hline F-test & $\mathbf{S}$ & $\mathbf{S}$ & $\mathrm{S}$ & $\mathbf{S}$ & $\mathbf{S}$ & $\mathrm{S}$ & $\mathbf{S}$ & $\mathbf{S}$ \\
\hline S. Ed. $( \pm)$ & 0.184 & 0.591 & 0.717 & 0.50 & 0.184 & 0.657 & 0.633 & 0.49 \\
\hline C.D. at 5\% & 0.379 & 1.219 & 1.480 & 1.03 & 0.379 & 1.356 & 1.307 & 1.01 \\
\hline
\end{tabular}

Table.5A Effect of flyash on water holding capacity (\%) of post-harvest soil under sunflower spinach sunflower crop rotation at $0-15 \mathrm{~cm}$ depth

\begin{tabular}{|c|c|c|c|c|c|c|c|c|}
\hline \multirow{2}{*}{ Treatment } & \multicolumn{5}{|c|}{$2015-16$} & \multicolumn{4}{c|}{ 2016-17 } \\
\cline { 2 - 10 } & Sunflower & Spinach & Sunflower & Mean & Sunflower & Spinach & Sunflower & Mean \\
\hline $\mathrm{T}_{1}$ & 63.39 & 62.40 & 61.56 & 62.45 & 63.30 & 62.35 & 61.15 & 62.27 \\
\hline $\mathrm{T}_{2}$ & 66.22 & 64.10 & 64.48 & 64.93 & 66.32 & 64.83 & 64.35 & 65.17 \\
\hline $\mathrm{T}_{3}$ & 67.22 & 65.25 & 65.60 & 66.02 & 67.26 & 66.28 & 65.38 & 66.31 \\
\hline $\mathrm{T}_{4}$ & 68.25 & 65.86 & 66.33 & 66.81 & 68.35 & 67.12 & 66.28 & 67.25 \\
\hline $\mathrm{T}_{5}$ & 69.21 & 66.45 & 66.93 & 67.53 & 69.31 & 67.99 & 67.01 & 68.10 \\
\hline $\mathrm{T}_{6}$ & 70.34 & 67.38 & 67.94 & 68.55 & 70.40 & 68.92 & 68.13 & 69.15 \\
\hline $\mathrm{T}_{7}$ & 71.55 & 68.59 & 69.24 & 69.79 & 71.56 & 70.55 & 69.20 & 70.44 \\
\hline F-test & $\mathbf{S}$ & $\mathbf{S}$ & $\mathrm{S}$ & $\mathbf{S}$ & $\mathbf{S}$ & $\mathrm{S}$ & $\mathbf{S}$ & $\mathbf{S}$ \\
\hline S. Ed. $\mathbf{(})$ & $\mathbf{0 . 4 0 4}$ & $\mathbf{0 . 5 8 6}$ & $\mathbf{0 . 5 3 9}$ & 0.51 & 0.371 & 0.605 & 0.347 & 0.44 \\
\hline C.D. at 5\% & $\mathbf{0 . 8 3 3}$ & $\mathbf{1 . 2 0 9}$ & $\mathbf{1 . 1 1 2}$ & 1.05 & 0.766 & 1.248 & 0.717 & 0.91 \\
\hline
\end{tabular}


Table.5B Effect of flyash on water holding capacity (\%) of post-harvest soil under sunflower spinach sunflower crop rotation at $15-30 \mathrm{~cm}$ depth

\begin{tabular}{|c|c|c|c|c|c|c|c|c|}
\hline Treatment & \multicolumn{4}{|c|}{ 2015-16 } & \multicolumn{2}{|r|}{1.248} & \multicolumn{2}{|l|}{ 2016-17 } \\
\hline & Sunflower & Spinach & Sunflower & Mean & Sunflower & Spinach & Sunflower & Mean \\
\hline $\mathrm{T}_{1}$ & 63.23 & 62.31 & 61.48 & 62.34 & 63.39 & 62.40 & 61.56 & 62.45 \\
\hline $\mathrm{T}_{2}$ & 66.07 & 63.97 & 64.35 & 64.80 & 66.22 & 64.10 & 64.48 & 64.93 \\
\hline $\mathrm{T}_{3}$ & 67.14 & 65.03 & 65.38 & 65.85 & 67.22 & 65.25 & 65.60 & 66.02 \\
\hline $\mathrm{T}_{4}$ & 68.12 & 65.81 & 66.28 & 66.74 & 68.25 & 65.86 & 66.33 & 66.81 \\
\hline $\mathrm{T}_{5}$ & 69.00 & 66.20 & 66.68 & 67.29 & 69.21 & 66.45 & 66.93 & 67.53 \\
\hline $\mathrm{T}_{6}$ & 70.20 & 67.24 & 67.80 & 68.41 & 70.34 & 67.38 & 67.94 & 68.55 \\
\hline $\mathrm{T}_{7}$ & 71.30 & 68.21 & 68.86 & 69.46 & 71.55 & 68.59 & 69.24 & 69.79 \\
\hline F-test & $\mathbf{S}$ & $\mathbf{S}$ & $\mathrm{S}$ & $\mathbf{S}$ & $\mathbf{S}$ & $\mathrm{S}$ & $\mathbf{S}$ & $\mathbf{S}$ \\
\hline S. Ed. ( $( \pm)$ & 0.401 & 0.604 & 0.604 & 0.54 & 0.404 & 0.586 & 0.539 & 0.51 \\
\hline C.D. at $5 \%$ & 0.829 & 1.247 & 1.246 & 1.11 & 0.833 & 1.209 & 1.112 & 1.05 \\
\hline
\end{tabular}

Figure.1A Effect of flyash on porosity (\%) of post-harvest soil under sunflower spinach sunflower crop rotation at 0-15 cm depth in 2015-16

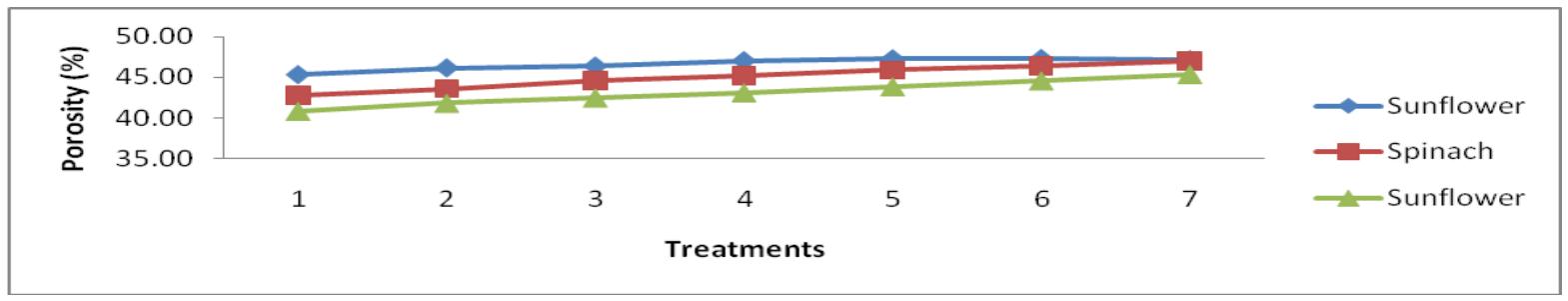

Figure.1B Effect of flyash on porosity (\%) of post-harvest soil under sunflower spinach sunflower crop rotation at 0-15 cm depth in 2016-17

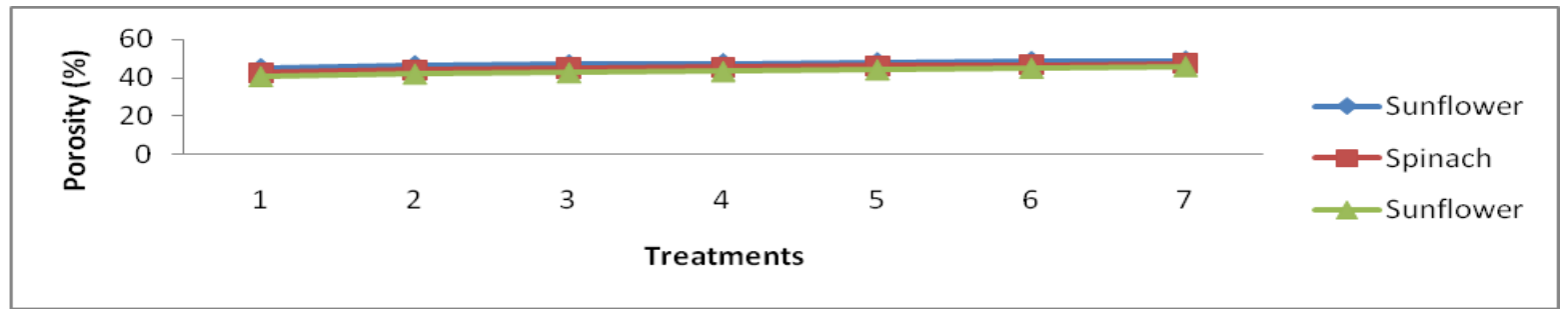

Figure.1C Effect of flyash on porosity (\%) of post-harvest soil under sunflower spinach sunflower crop rotation at 15-30 cm depth in 2015-16

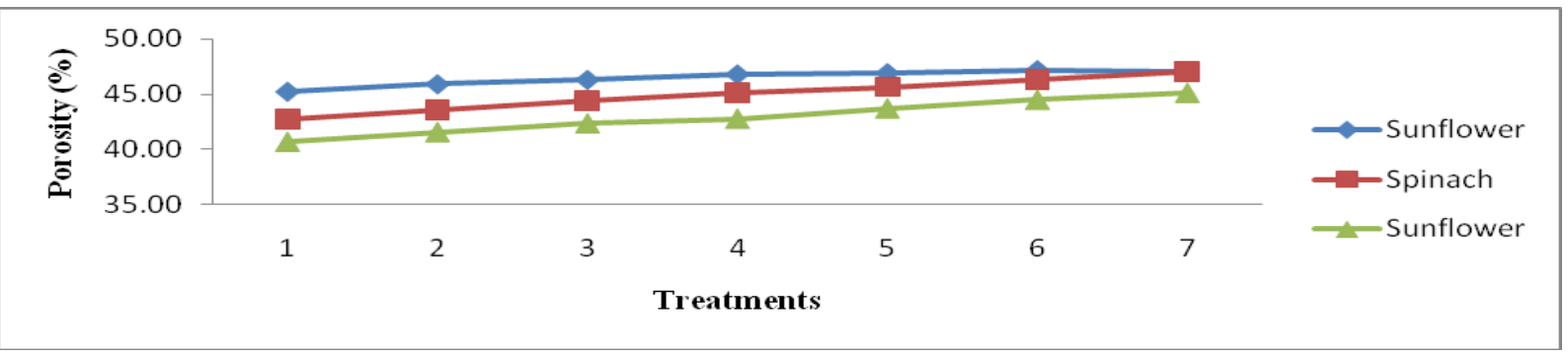


Figure.1D Effect of flyash on porosity (\%) of post-harvest soil under sunflower spinach sunflower crop rotation at 15-30 cm depth in 2016-17

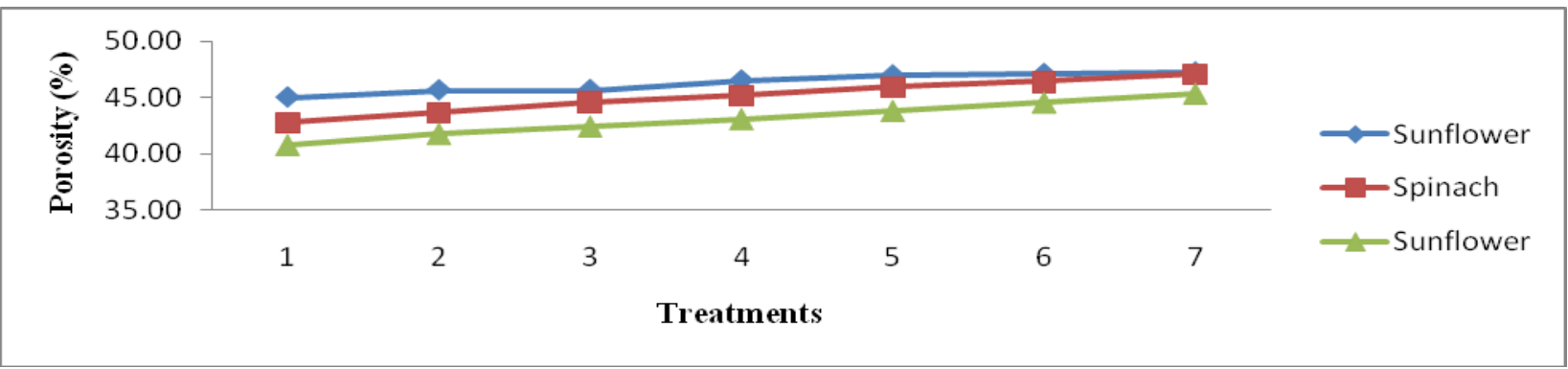

Figure.2A Effect of Flyash on Bulk Density $\left(\mathrm{mg} / \mathrm{m}^{3}\right)$ of Post-Harvest Soil under Sunflower Spinach Sunflower Crop rotation at 0-15 cm depth in 2015-16

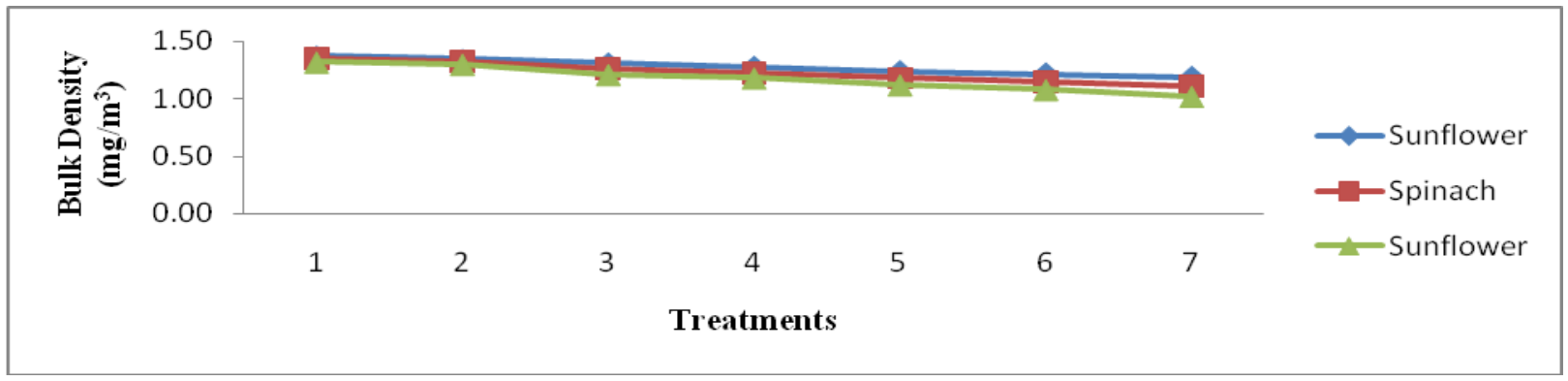

Figure.2B Effect of flyash on bulk density $\left(\mathrm{mg} / \mathrm{m}^{3}\right)$ of post-harvest soil under sunflower spinach sunflower crop rotation at $0-15 \mathrm{~cm}$ depth in 2016-17

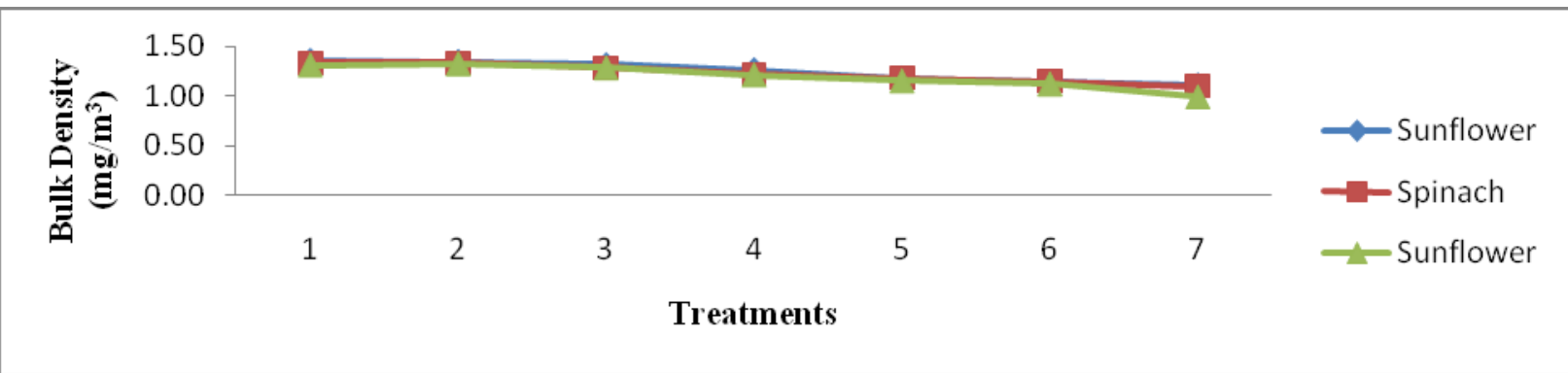

Figure.2C Effect of flyash on bulk density $\left(\mathrm{mg} / \mathrm{m}^{3}\right)$ of post-harvest soil under sunflower spinach sunflower crop rotation at 15-30 cm depth in 2015-16

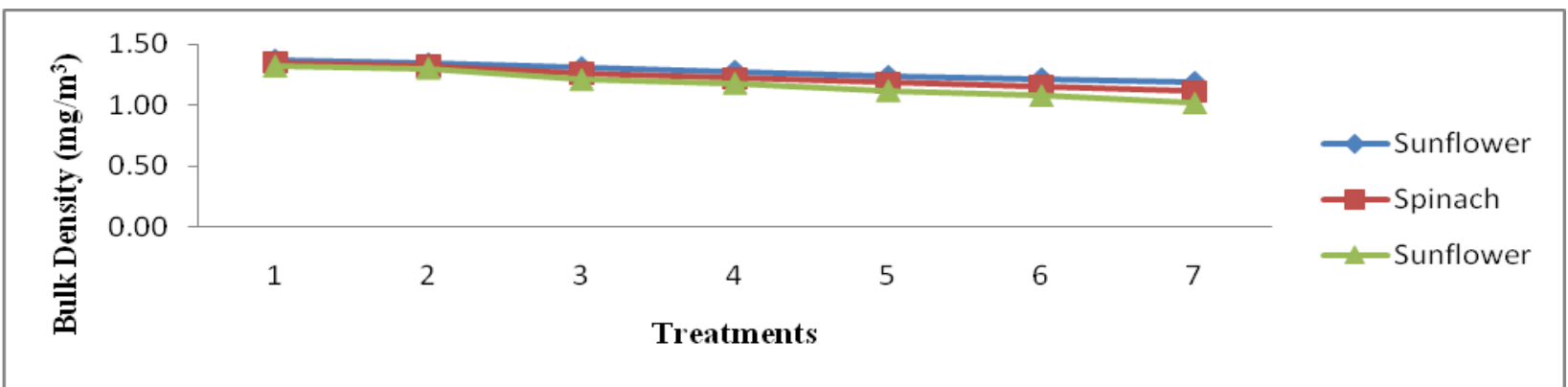


Figure.2D Effect of flyash on bulk density $\left(\mathrm{mg} / \mathrm{m}^{3}\right)$ of post-harvest soil under sunflower spinach sunflower crop rotation at $15-30 \mathrm{~cm}$ depth $2016-17$ oil particle density $\left(\mathrm{mg} / \mathrm{m}^{3}\right)$

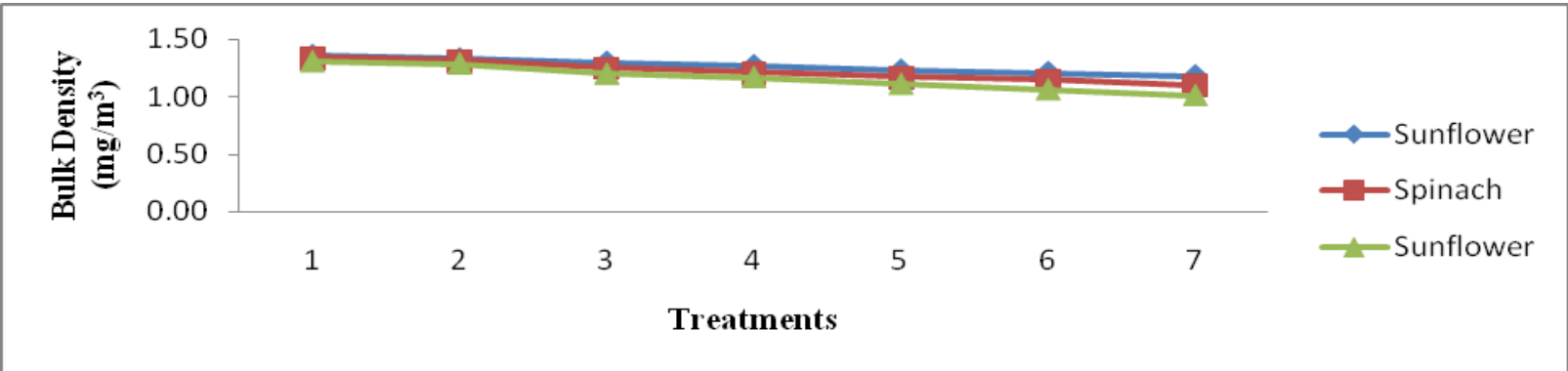

Figure.3A Effect of flyash on particle density $\left(\mathrm{mg} / \mathrm{m}^{3}\right)$ of post-harvest soil under sunflower spinach sunflower crop rotation at 0-15 cm depth in 2015-16

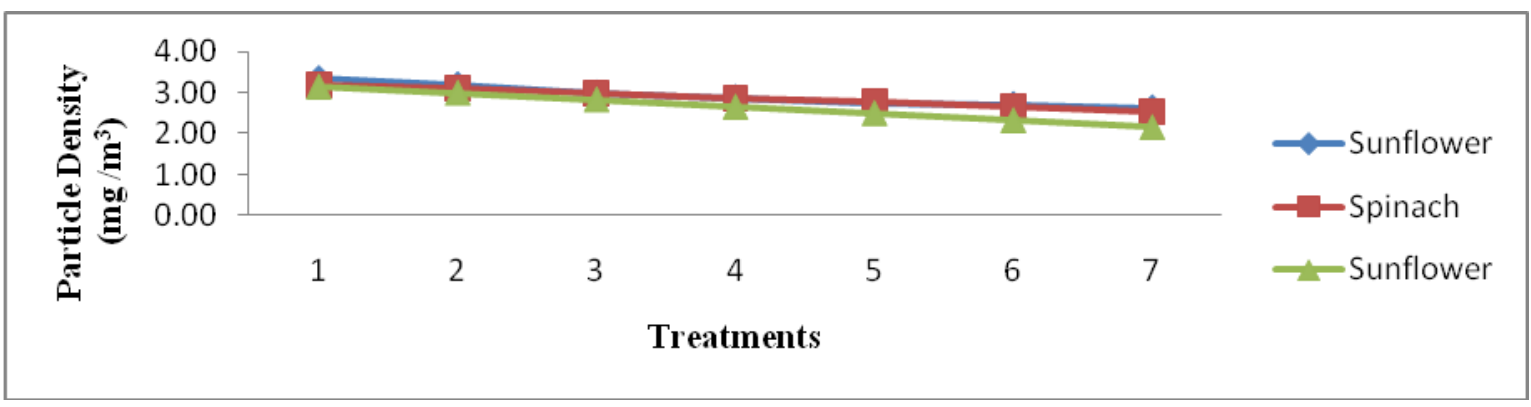

Figure.3B Effect of flyash on particle density $\left(\mathrm{mg} / \mathrm{m}^{3}\right)$ of post-harvest soil under sunflower spinach sunflower crop rotation at 0-15 cm depth in 2016-17

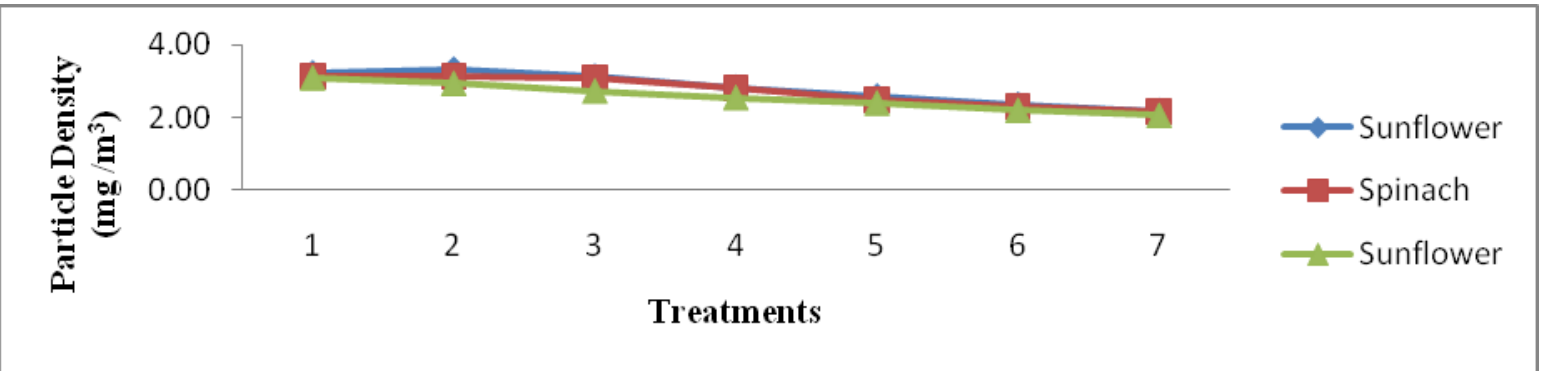

Figure.3C Effect of flyash on particle density $\left(\mathrm{mg} / \mathrm{m}^{3}\right)$ of post-harvest soil under sunflower spinach sunflower crop rotation at 15-30 cm depth 2016-17

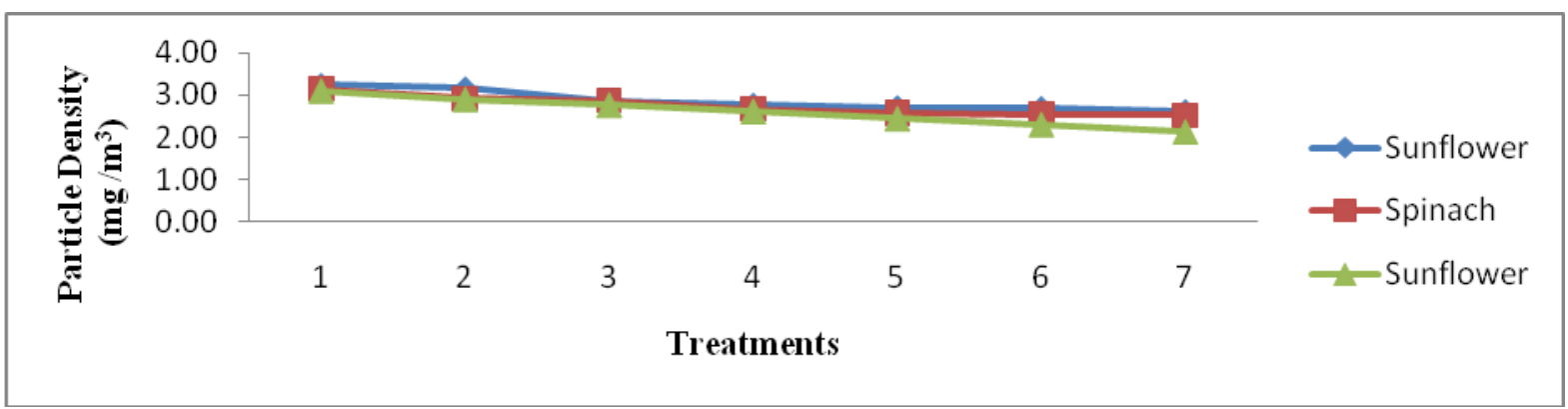


Figure.3D Effect of flyash on particle density $\left(\mathrm{mg} / \mathrm{m}^{3}\right)$ of post-harvest soil under sunflower spinach sunflower crop rotation at 15-30 cm depth in 2016-17

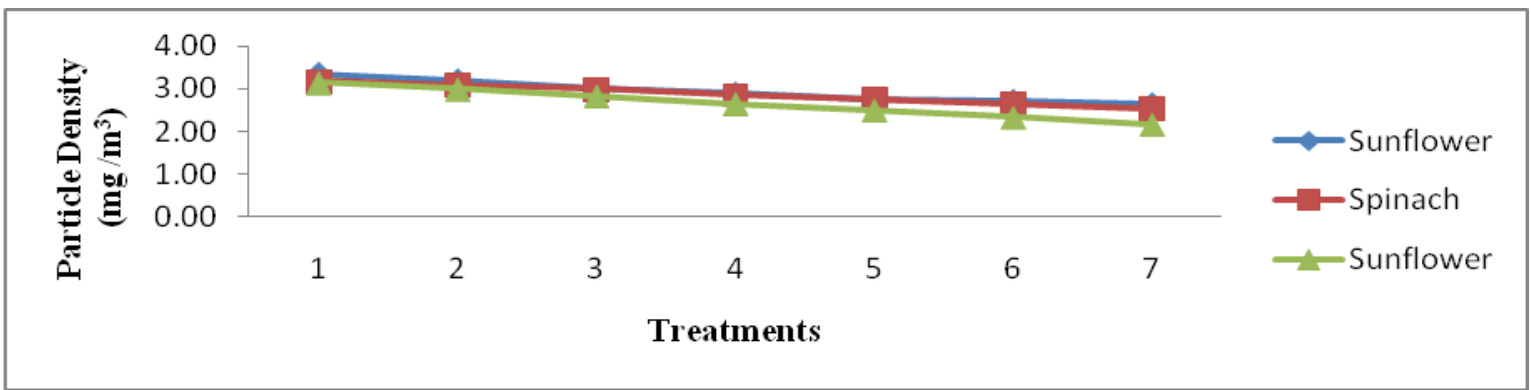

Figure.4A Effect of flyash on solid (\%) of post-harvest soil under sunflower spinach sunflower crop rotation at $0-15 \mathrm{~cm}$ depth in 2015-16

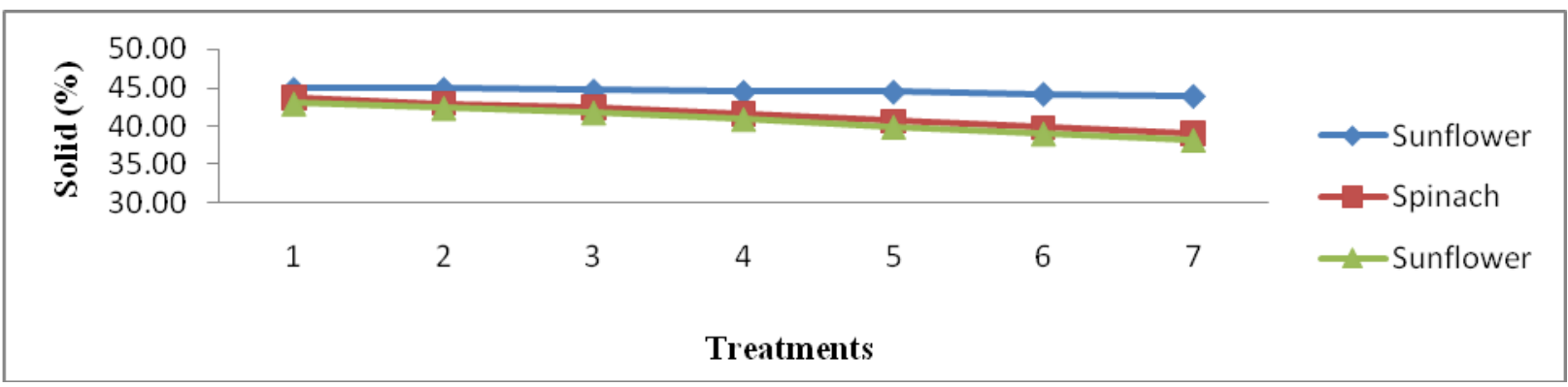

Figure.4B Effect of flyash on solid (\%) of post-harvest soil under sunflower spinach sunflower crop rotation at $0-15 \mathrm{~cm}$ depth in 2016-17

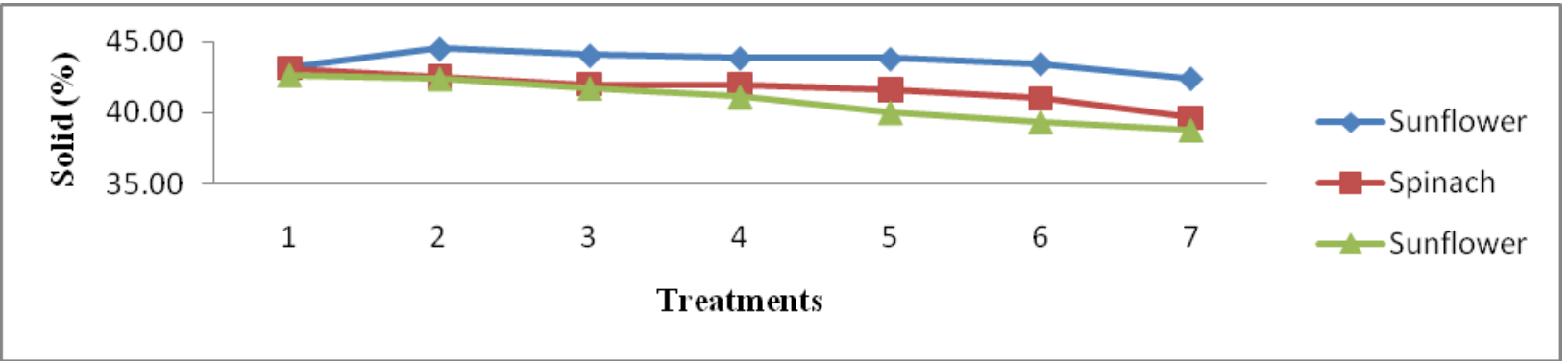

Figure.4C Effect of flyash on solid (\%) of post-harvest soil under sunflower spinach sunflower crop rotation at 15-30 $\mathrm{cm}$ depth in 2015-16

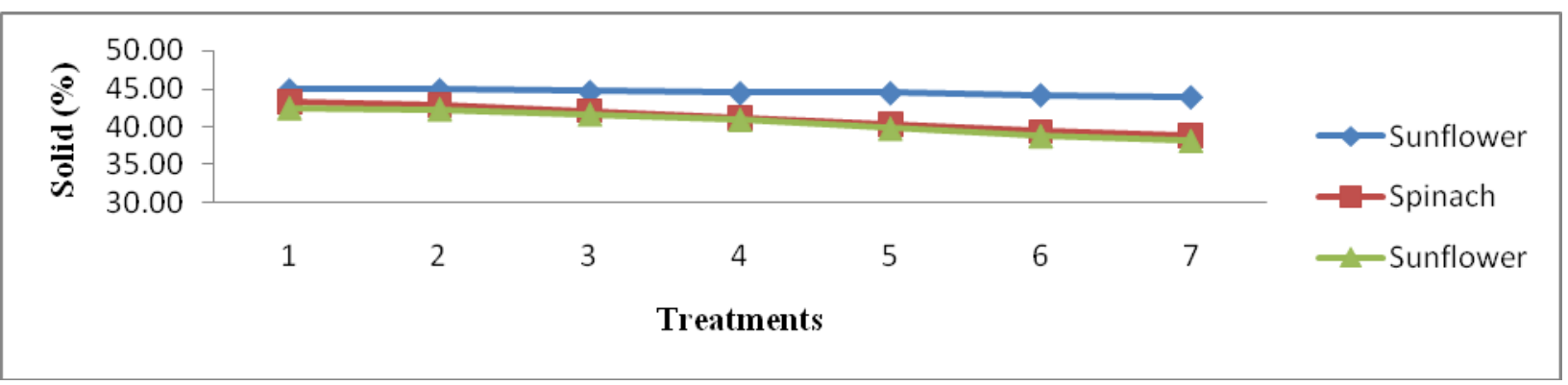


Figure.4D Effect of flyash on solid (\%) of post-harvest soil under sunflower spinach sunflower crop rotation at 15-30 $\mathrm{cm}$ depth in 2016-17

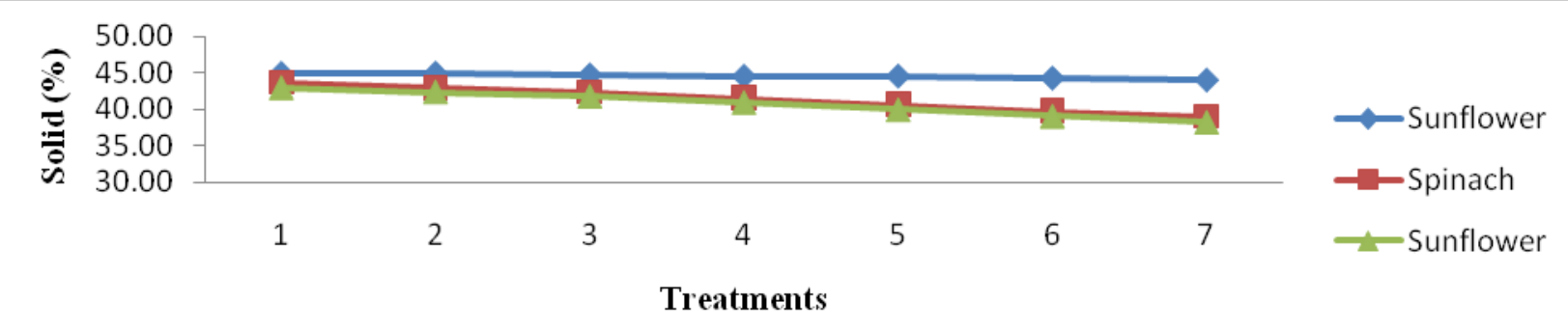

Figure.5A Effect of Flyash on Water Holding Capacity (\%) of Post Harvest Soil under Sunflower Spinach Sunflower Crop rotation at 0-15 cm depth in 2015-16

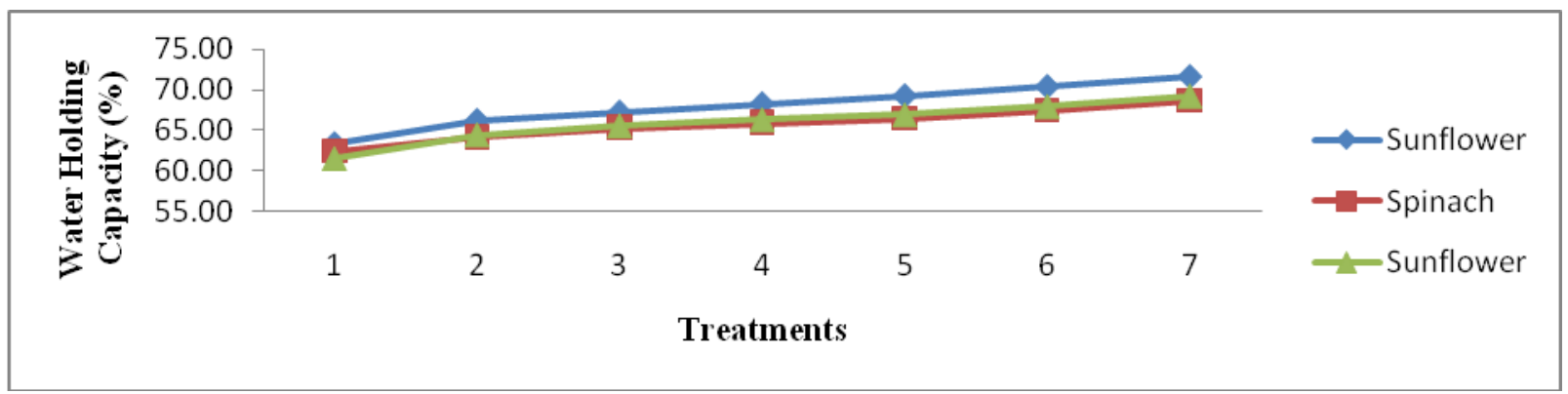

Figure.5B Effect of flyash on water holding capacity (\%) of post harvest soil under sunflower spinach sunflower crop rotation at 0-15 cm depth in 2016-17

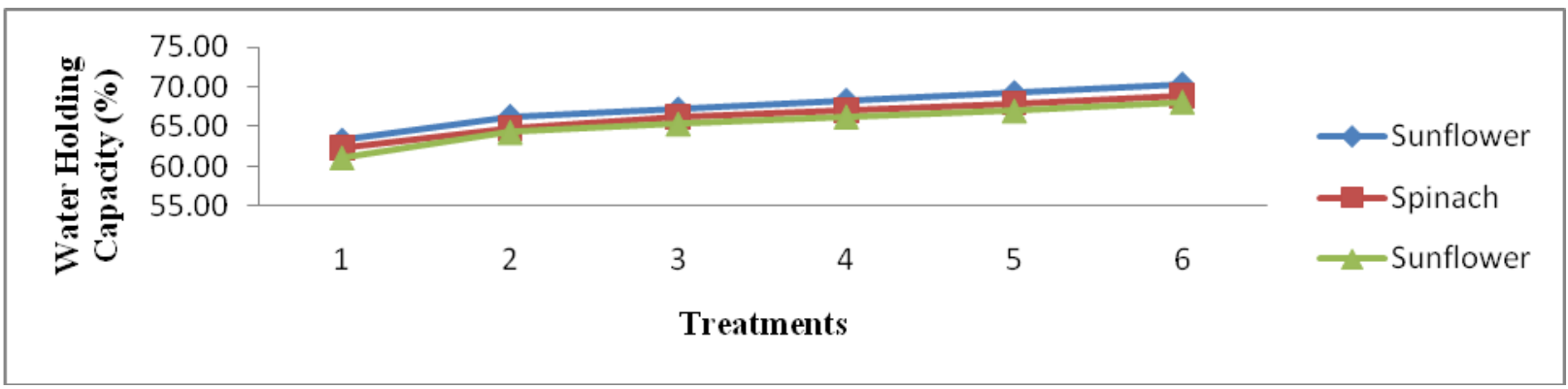

Figure.5C Effect of flyash on water holding capacity (\%) of post harvest soil under sunflower spinach sunflower crop rotation at 15-30 cm depth in 2015-16

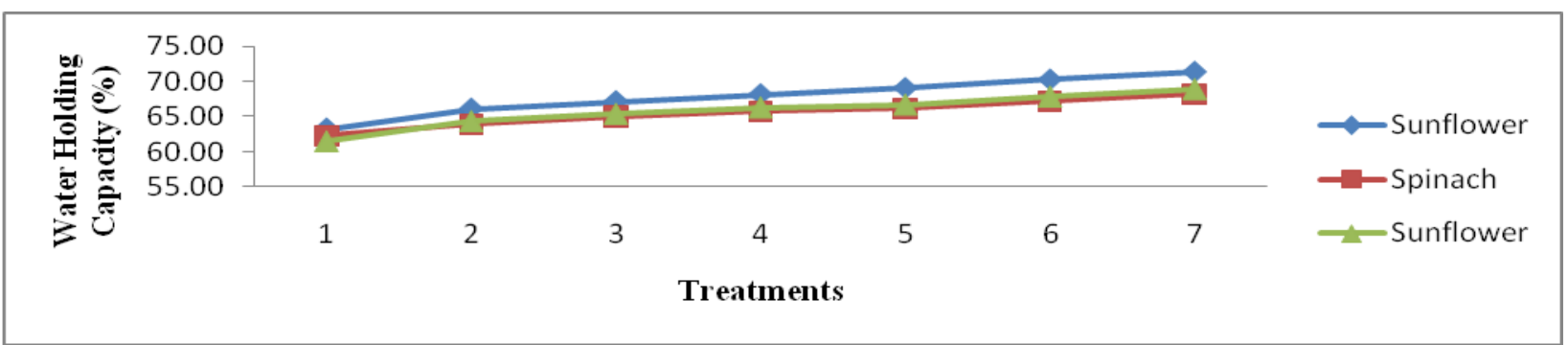


Figure.5D Effect of fly ash on water holding capacity (\%) of post-harvest soil under sunflower spinach sunflower crop rotation at 15-30 cm depth in 201

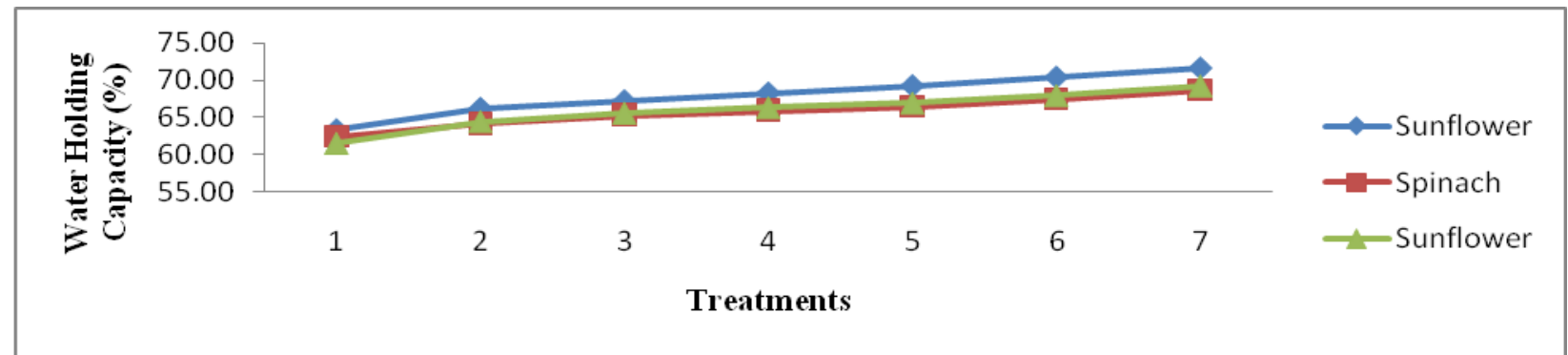

The maximum on soil solid space $43.83(\%)$ and $42.98(\%)$ was found in $\mathrm{T}_{1}$ (Control) at $0-15 \mathrm{~cm}$ soil depth which was statistically at par with the $\mathrm{T}_{2}$ in 2015-16 and 2016-17 and minimum on solid space $40.37(\%)$ and $40.28(\%)$ was found in $\mathrm{T}_{7}$ (Flyash@50 metric tons ha $^{-1}+\mathrm{RDF}$ ). It was found that the soil solid space was decreased increased dose of flyash in each treatment and also decreases the on soil solid space after harvesting of each crop under crop rotation in both experimental years. The maximum on soil solid space $43.51(\%)$ and $43.83(\%)$ was found in $\mathrm{T}_{1}$ (Control) at $15-30$ $\mathrm{cm}$ soil depth which was statistically at par with the $T_{2}$ in 2015-16 and 2016-17 and minimum on soil solid space $40.28 \quad\left(\mathrm{mg} / \mathrm{m}^{3}\right)$ and $40.37\left(\mathrm{mg} / \mathrm{m}^{3}\right.$ ) was found in $\mathrm{T}_{7}$ (Flyash @ 50 metric tons $\left.\mathrm{ha}^{-1}+\mathrm{RDF}\right)$. It was found that the soil solid space was decreased as the dose of flyash increases in each treatment and also decreases the on soil solid space after harvesting of each crop under crop rotation in both experimental years.

\section{Soil water holding capacity $(\%)$}

The tables of 4(a) and (b) and figures of 4(a), (b), (c) and (d) shows that the effect of flyash on soil water holding capacity (\%) of postharvest soil under sunflower-spinach-sunflower crop rotation at $0-15 \mathrm{~cm}$ and $15-30 \mathrm{~cm}$ soil depth was found satisfactory in both experimental years 2015-16 and 2016-17. The maximum on soil water holding capacity 69.24(\%) and $70.44(\%)$ was found in $\mathrm{T}_{7}$ (Flyash@ 50 metric tons ha ${ }^{-1}+\mathrm{RDF}$ ) at $0-15$ $\mathrm{cm}$ soil depth which was statistically at par with the $\mathrm{T}_{2}$ in 2015-16 and 2016-17 and minimum on soil water holding capacity $62.45(\%)$ and 62.27 (\%) was found in $\mathrm{T}_{1}$ (Control). It was found that the soil water holding capacity was increases with the increased dose of flyash in each treatment and also observed that decreases the on soil water holding capacity after harvesting of each crop under crop rotation in both experimental years. The maximum on soil water holding capacity $69.46(\%)$ and $69.79(\%)$ was found in $\mathrm{T}_{7}$ (Flyash @ 50 metric tons $\mathrm{ha}^{-1}+$ RDF) at $15-30 \mathrm{~cm}$ soil depth which was statistically at par with the $\mathrm{T}_{2}$ in 2015-16 and 2016-17 and minimum on soil water holding capacity $62.34(\%)$ and $62.45(\%)$ was found in $\mathrm{T}_{1}$ (Control). It was found that the soil water holding capacity was increases with the increased dose of flyash in each treatment and also observed that decreases the on soil water holding capacity after harvesting of each crop under crop rotation in both experimental years. Fly-ash generally decreased the bulk density of soils leading to improved soil porosity, workability and enhanced water-retention capacity (Page et al., 1979). The results revealed that the increase in fly-ash concentration in the soil $(0 \%, 10 \%$, and $20 \%$ up to $50 \%$ ) increase water-holding capacity Similar results obtained by Khan and Khan (1996) and Singh and Siddiqui (2003).

In conclusion, the findings of present study conclude that impact in control soil and fly ash admixed soil at different treatment level varied markedly. Addition of fly ash in soil improved the soil physical properties like bulk density, particle density, water holding capacity 
(W.H.C.) and porosity. The concentration of major nutrients such as nitrogen, phosphorus and potassium are slightly increased in treated soils as compared to control. Soil applications to fly ash at lower levels were found to be beneficial for the plant growth in the present study. However, at higher application levels of fly ash, reduction in growth and yield of sunflower and spinach were observed. It was found that the $\mathrm{T}_{5}$ (Flyash @ 30 metric tons ha $\left.{ }^{1}+\mathrm{RDF}\right)$ gives the high growth and yield for the sunflower and spinach. The present study showed significant increase in yields as well as biomass without any adverse effect on soil health or crop and the presence of heavy metal is too low to make any harmful impact.

\section{References}

Aitken, R. L., Campbell, D. J. and Bell, L. C.(1984) Properties of Australian fly ash relevant to their agronomic utilization, Aust. J. Soil Res., Vol. 22, pp. 443-453.

Bhandari, K. (2006) Studies on the Effect of Fly Ash and Plant Hormones Treated Soil in the Increased Protein and Amino Acid Content in the Seeds Ground Nut, Asian Journal of Chemistry, Vol. 20, p p. 15.

Bozkurt, M. A. and Karacal, I. (2001) Quantitative Relationship between Nutrient Contents and Oil Quality of Sunflower Seed, Journal of Food and Science Technology, Vol. 38(6), pp. 635-638. Brighton Crop Protection Conference, pp. 658.

Chadha, Y. R. (1998) Lime Requirement for Proper Growth of Ground Nut, Wealth of India," CSIR Publication, New Delhi, pp. 90-102.

Chang, A. C., Lund, L. J. and Page, A. L. (1977) Physical properties of flyash amended soils. J Environ Qual, Vol. 6, pp. 267-70.

Change, C., Lund, L. J., Page, A. L. and Warneke,
J. E. (1979) Physical Properties of Fly Ash Amended Soil, Journal Environment Quality, Vol. 6(3), pp. 267-270.

Garg, R. N., Pathak, H. and Das, D. K., (2005) Use of flyash and biogas slurry for improving wheat yield and physical properties of soil. Enviro Monit Assess, Vol. 107, pp. 1-9.

Kene, D. R., Lanjewar, S. A. and Ingole, B. M. (1991) Effect of application of flyash on physico-chemical properties of soils. J Soils Crops, Vol. 1(1), pp. 11-18.

Pandey, V. C., Abhilash, P. C., Upadhyay, R. N. and Tiwari, D. D. (2009) Application of fly ash on the growth performance and translocation of toxic heavy metals within Cajanus cajan L. implication for safe utilization of fly ash for agricultural production, Journal of Hazardous Materials, Vol. (166), pp. 255-259.

Page, A. L., Elseewi, A. A. and Straughan, I.(1979) Physical and chemical properties of fly ash from coal fired power plants with reference to environmental impacts, Residue Rev., Vol. 71, pp. 82-120.

Pathan S.M., Aylmore L.A.G., and Colmer T.D. (2001) Soil properties and turf growth on a sandy soil amended with flyash, plant Soil, Vol. 256, pp. 103-110

Raj, S. and Mohan, S. (2014) Approach for Improved Plant Growth Using Fly Ash Amended Soil, International Journal of Emerging Technology and Advanced Engineering, Vol. 4(6), pp. 709-715.

Ray, S. N. C., Ranjan, N., Kumari, K. and Sinha, R. C.(2003) International Journal of Engineering Research and Applications (IJERA) Vol. 2(5) pp. 193-203.

Siddhiqui, A. and Singh, L. P. (2003) Effect of fly ash on growth and yield of three cultivars of rice, Bioresource Technology, Vol. 86(1), 73-78.

\section{How to cite this article:}

Nouraldin Almahdi Ibrahim Basha, Abhishek James, Ram Bharose and Smriti Rao, P. 2018. Impact of Flyash on Soil physical Properties under Sunflower-Spinach-Sunflower Crop Rotation System in Central India. Int.J.Curr.Microbiol.App.Sci. 7(12): 1815-1828. doi: https://doi.org/10.20546/ijcmas.2018.712.212 